\title{
On Minimal Triangulations of Products of Convex Polygons
}

\author{
Michelle Bucher-Karlsson
}

Received: 13 September 2007 / Revised: 14 May 2008 / Accepted: 25 May 2008 /

Published online: 21 June 2008

(C) Springer Science+Business Media, LLC 2008

\begin{abstract}
We give new lower bounds for the minimal number of simplices needed in a triangulation of the product of two convex polygons, improving the lower bounds in Bowen et al. (Topology 44:321-339, 2005).
\end{abstract}

Keywords Minimal triangulations - Convex polytopes

\section{Introduction}

The use of volume of simplices in hyperbolic geometry for combinatorial problems, initiated by Thurston, has proven successful for minimal triangulations of polytopes. For example, embedding an $n$-gon ideally in the hyperbolic plane, it is elementary to see, since the area of a geodesic triangle is majorized by the (constant) area of an ideal geodesic triangle, that the minimal number of 2-dimensional simplices needed for a triangulation of a convex $n$-gon is equal to $n-2$. Similarly, the 3 -cube can be embedded ideally in the 3-dimensional hyperbolic space in such a way that its triangulation in 5 simplices consists of regular ideal simplices. As regular simplices have maximum volume, this shows that the triangulation is minimal. More generally, Smith [5] gives the best lower bound so far for the minimum number of $n$-dimensional simplices in a triangulation of the $n$-cube as the ratio of the hyperbolic volume of the ideal cube to the ideal regular simplex in hyperbolic $n$-space. Also, Sleator et al. [4] relate the hyperbolic volume of simplices to the size of minimal triangulations of polytopes and balls and use this relation to compute the asymptotic combinatorial diameter of the Stasheff polytope (or associahedron).

Support from the Göran Gustafsson Foundation and the Swedish Research Council (VR) grant 621-2007-6250 is gratefully acknowledged.

M. Bucher-Karlsson ( $₫)$

Math. Dept., KTH, 10044 Stockholm, Sweden

e-mail: mickar@math.kth.se

url: http://www.math.kth.se/ mickar/ 
Here, we will use the cocycle $\mathrm{Vol}_{4}$, which is cohomologous to the volume form on the product of two copies of the hyperbolic plane, to give new lower bounds on the minimal number $T(m, n)$ of top dimensional simplices in a triangulation of the product $P(m) \times P(n)$, where $P(m)$ denotes the convex polygon with $m$ vertices. Note that all our triangulations of polytopes are supposed not to have other vertices than those of the original polytope. Previously known are the lower bounds $T(m, n) \geq$ $2 m n-(8 / 3)(m+n)$ and $T(m, 4) \geq 7.5 m-3$ obtained in [1]. We prove the following:

Theorem $1 T(m, n) \geq 3.125 \cdot m n-5(m+n)+6$.

Theorem $2 T(m, 4) \geq \frac{23}{3} m-\frac{44}{3}$.

Thus, we are coming closer to the upper bounds $T(m, n) \leq 3.5 m n-6(m+n)+8$ and $T(m, 4) \leq 8 m-16$ established in [1] for even $m$ and $n$. The upper bounds for odd $m$ and $n$ are slightly higher.

Since the arguments used in our proofs are of homological type, our method further gives the same lower bounds for the polytopal Gromov norm $\|P(m) \times P(n)\|$ of $P(m) \times P(n)$ (for a definition, see [1]), for which the upper bound $\|P(m) \times P(m)\| \leq$ $3.25 m^{2}-8.5 m$ is known from [1] for even $m$. Those lower bounds confirm the conjecture in [1] that the polytopal Gromov norm of $P(m) \times P(n)$ is indeed closer to the author's upper bound than to their lower bound of $2 m n+O(m+n)$ but it contradicts the author's guess that it may behave as $3 m n+O(m+n)$.

Our lower bounds are only sharp for incidental low values of $m$ and $n$. If $m$ or $n$ is odd, then our proof could, with some care, be improved to give slightly better lower bounds. Observe also that for $n=3$, the value of $T(m, 3)$ is known and computed in [1].

The combinatorial volume cocycle $\mathrm{Vol}_{4}$ used for the proofs of Theorems 1 and 2 already appears in [2]: Its sup norm $\left\|\mathrm{Vol}_{4}\right\|_{\infty}=2 / 3$ as a cohomology class in $H_{c}^{4}(P S L(2, \mathbb{R}) \times P S L(2, \mathbb{R}))$ relates the simplicial volume of products of surfaces to the simplicial volume of their factors:

$$
\left\|\Sigma_{g} \times \Sigma_{h}\right\|=\frac{3}{2}\left\|\Sigma_{g}\right\|\left\|\Sigma_{h}\right\|=24(g-1)(h-1),
$$

where $\Sigma_{g}$ and $\Sigma_{h}$ are surfaces of genus $g \geq 1$ and $h \geq 1$, respectively. This is the first product formula for the simplicial volume and the first example of an exact value of a nonvanishing simplicial volume for a manifold not admitting a constant curvature metric. We refer to [2] for more details.

This paper is structured as follows: In Sect. 2, we define several combinatorial volume cocycles for certain low-dimensional polytopes $P$ and see that they define cohomology classes in the relative cohomology $H^{*}(P, \partial P)$. In Sect. 3, we compute the minimal number of 3 -simplices in prisms $P(m) \times[0,1]$, a result which already is known from [3]. In Sect. 4, we give a (probably sharp) bound on the number of 4simplices in a triangulation of $P(m) \times P(n)$ having at least one 3-face in the boundary $\partial(P(m) \times P(n))$. And finally, in Sect. 5, we use the combinatorial volume cocycle $\mathrm{Vol}_{4}$ in order to estimate the number of remaining (interior) 4-simplices in such a triangulation. 


\section{Combinatorial Volume Cocycles}

\subsection{Cocycles on Products of Low-Dimensional Spheres}

We identify the 0 -dimensional sphere $S^{0}$ with two endpoints 0 and 1 of the unit interval $[0,1]$ and define a cochain

$$
v:\left(S^{0}\right)^{2} \longrightarrow\{-1,0,+1\}
$$

as

$$
\begin{aligned}
& v(0,0)=v(1,1)=0, \\
& v(0,1)=+1, \\
& v(1,0)=-1 .
\end{aligned}
$$

Observe that $v$ is alternating by definition. Furthermore, $v$ is a cocycle, i.e., it satisfies the cocycle relation $\delta v(x, y, z)=v(y, z)-v(x, z)+v(x, y)=0$ for all $x, y, z$ in $S^{0}$.

Fix now an orientation on the circle $S^{1}$ and recall that the orientation cocycle Or is defined as

$$
\text { Or: } \begin{aligned}
&\left(S^{1}\right)^{3} \longrightarrow \mathbb{R}, \\
&\left(x_{0}, x_{1}, x_{2}\right) \longmapsto \begin{cases}+1 & \text { if } x_{0}, x_{1}, x_{2} \text { are positively oriented, } \\
-1 & \text { if } x_{0}, x_{1}, x_{2} \text { are negatively oriented, } \\
0 & \text { if } x_{i}=x_{j} \text { for } i \neq j .\end{cases}
\end{aligned}
$$

Note that the cochain Or is alternating by definition. To check that it is a cocycle, we verify that $\delta \operatorname{Or}\left(x_{0}, \ldots, x_{3}\right)=0$ for any 4 -tuple of points $x_{0}, \ldots, x_{3}$ in $S^{1}$. Let us first assume that the points are all distinct. Since $\delta$ Or is alternating, we can without loss of generality assume that the points $x_{0}, \ldots, x_{3}$ are positively cyclically ordered. In particular, $\operatorname{Or}\left(x_{0}, \ldots, \widehat{x}_{i}, \ldots, x_{3}\right)=+1$ for every $i$, and

$$
\delta \operatorname{Or}\left(x_{0}, \ldots, x_{3}\right)=\sum_{i=0}^{3}(-1)^{i} \operatorname{Or}\left(x_{0}, \ldots, \widehat{x}_{i}, \ldots, x_{3}\right)=1-1+1-1=0 .
$$

If the four points $x_{0}, \ldots, x_{3}$ are not all distinct, then we can assume that $x_{0}=x_{1}$ and we have

$$
\delta \operatorname{Or}\left(x_{0}, \ldots, x_{3}\right)=\operatorname{Or}\left(x_{1}, x_{2}, x_{3}\right)-\operatorname{Or}\left(x_{0}, x_{2}, x_{3}\right)+0-0=0 .
$$

The cup product of the orientation cocycle with $v$ is given by

$$
\text { Or } \cup v: \quad \begin{aligned}
\left(S^{1} \times S^{0}\right)^{4} & \longrightarrow \mathbb{R}, \\
\left(\left(x_{0}, y_{0}\right), \ldots,\left(x_{3}, y_{3}\right)\right) & \longmapsto \operatorname{Or}\left(x_{0}, x_{1}, x_{2}\right) \cdot v\left(y_{2}, y_{3}\right) .
\end{aligned}
$$

Observe that, in view of the well-known and straightforward formula

$$
\delta(\operatorname{Or} \cup v)=\delta \operatorname{Or} \cup v-\operatorname{Or} \cup \delta v=0,
$$


the cochain Or $\cup v$ is also a cocycle. However, it is clearly not alternating, so that we define a cochain

$$
\mathrm{Vol}_{3}:\left(S^{1} \times S^{0}\right)^{4} \longrightarrow \mathbb{R}
$$

as the alternation of $\operatorname{Or} \cup v$ :

$$
\operatorname{Vol}_{3}\left(z_{0}, \ldots, z_{3}\right)=\frac{1}{4 !} \sum_{\sigma \in S y m(4)} \operatorname{sign}(\sigma)(\operatorname{Or} \cup v)\left(z_{\sigma(0)}, \ldots, z_{\sigma(3)}\right)
$$

for every 4-tuple $\left(z_{0}, \ldots, z_{3}\right)$ in $\left(S^{1} \times S^{0}\right)^{4}$. Observe that

$$
\text { Or } \cup v-\mathrm{Vol}_{3}=\delta b_{3},
$$

where $b_{3}:\left(S^{1} \times S^{0}\right)^{3} \rightarrow \mathbb{R}$ is the cochain defined as

$$
b_{3}\left(\left(x_{0}, y_{0}\right),\left(x_{1}, y_{1}\right),\left(x_{2}, y_{2}\right)\right)=\frac{1}{3} \operatorname{Or}\left(x_{0}, x_{1}, x_{2}\right) \cdot\left(v\left(y_{1}, y_{2}\right)+v\left(y_{0}, y_{2}\right)\right) \text {. }
$$

In particular, the cochain $\mathrm{Vol}_{3}$ also is a cocycle.

The cup product of the orientation cocycle with itself is given by

$$
\text { Or } \begin{aligned}
\left(S^{1} \times S^{1}\right)^{5} & \longrightarrow \mathbb{R} \\
\left(\left(x_{0}, y_{0}\right), \ldots,\left(x_{4}, y_{4}\right)\right) & \longmapsto \operatorname{Or}\left(x_{0}, x_{1}, x_{2}\right) \cdot \operatorname{Or}\left(y_{2}, y_{3}, y_{4}\right),
\end{aligned}
$$

and it is a cocycle in view of the formula

$$
\delta(\text { Or } \cup \text { Or })=\delta \text { Or } \cup \text { Or }+ \text { Or } \cup \delta \text { Or }=0 .
$$

We define the cochain

$$
\mathrm{Vol}_{4}:\left(S^{1} \times S^{1}\right)^{5} \longrightarrow \mathbb{R}
$$

as the alternation of Or $\cup$ Or:

$$
\operatorname{Vol}_{4}\left(z_{0}, \ldots, z_{4}\right)=\frac{1}{5 !} \sum_{\sigma \in \operatorname{Sym}(5)} \operatorname{sign}(\sigma)(\text { Or } \cup \text { Or })\left(z_{\sigma(0)}, \ldots, z_{\sigma(4)}\right)
$$

for every 5-tuple $\left(z_{0}, \ldots, z_{4}\right)$ in $\left(S^{1} \times S^{1}\right)^{5}$. Here also, Or $\cup$ Or and $\mathrm{Vol}_{4}$ differ by a coboundary. Indeed, letting $b_{4}:\left(S^{1} \times S^{1}\right)^{4} \rightarrow \mathbb{R}$ be the cochain

$$
\begin{aligned}
b_{4}\left(\left(x_{0}, y_{0}\right), \ldots,\left(x_{3}, y_{3}\right)\right)= & \frac{1}{3} \operatorname{Or}\left(x_{1}, x_{2}, x_{3}\right)\left(\operatorname{Or}\left(x_{1}, x_{3}, x_{4}\right)+\operatorname{Or}\left(x_{2}, x_{3}, x_{4}\right)\right) \\
& +\frac{1}{12}\left(\operatorname{Or}\left(x_{1}, x_{2}, x_{4}\right)\left(\operatorname{Or}\left(x_{1}, x_{3}, x_{4}\right)+\operatorname{Or}\left(x_{2}, x_{3}, x_{4}\right)\right)\right. \\
& +\operatorname{Or}\left(x_{1}, x_{3}, x_{4}\right)\left(-\operatorname{Or}\left(x_{1}, x_{2}, x_{4}\right)+\operatorname{Or}\left(x_{2}, x_{3}, x_{4}\right)\right) \\
& \left.+\operatorname{Or}\left(x_{2}, x_{3}, x_{4}\right)\left(-\operatorname{Or}\left(x_{1}, x_{2}, x_{4}\right)-\operatorname{Or}\left(x_{1}, x_{3}, x_{4}\right)\right)\right),
\end{aligned}
$$

one can check that $\mathrm{Or} \cup \mathrm{Or}-\mathrm{Vol}_{4}=\delta b_{4}$. Note that, in particular, $\mathrm{Vol}_{4}$ is a cocycle. 


\subsection{Some Homological Algebra}

Let $P$ be a polyhedral subset of $\mathbb{R}^{n}$, that is, a finite union of convex polytopes, and let

$$
C_{q}(P)=\left\{\sum_{\text {finite }} a_{\sigma} \sigma \mid a_{\sigma} \in \mathbb{R}, \sigma: \Delta^{q} \rightarrow P \text { affine, vertices of } \sigma \subset P^{0}\right\}
$$

be the real vector space over the basis of affine simplices $\sigma: \Delta^{q} \rightarrow P$, where $\Delta^{q}$ denotes the standard $q$-simplex mapping the vertices of $\Delta^{q}$ to the vertices $P^{0}$ of $P$. The relative chain complex of $(P, \partial P)$ is given, in degree $q$, by

$$
C_{q}(P, \partial P)=C_{q}(P) / C_{q}(\partial P) .
$$

The boundary operator $\partial: C_{q}(P) \rightarrow C_{q-1}(P)$ induces a boundary operator on the quotient $C_{*}(P, \partial P)$, and the resulting homology group $H_{*}(P, \partial P)$ is the relative homology of $(P, \partial P)$.

Any triangulation $T$ of the $n$-dimensional polytope $P$ determines in a unique (after the choice of an orientation on $P$ ) and natural way an affine cycle $z_{T}$ in $C_{n}(P, \partial P)$, which in turn gives a relative homology class

$$
\left[z_{T}\right] \in H_{n}(P, \partial P) .
$$

(All our triangulations are assumed not to have other vertices than those of $P$.) If $z_{1}, z_{2}$ are two affine cycles arising from two triangulations of $P$, then $\left[z_{1}\right]=\left[z_{2}\right]$ in $H_{n}(P, \partial P)$. To prove that, one can either use some simple homological argument involving the Poincaré duality, as done at the end of this section, or exhibit chains $c$ in $C_{n+1}(P)$ and $b$ in $C_{n}(\partial P)$ such that

$$
z_{2}-z_{1}=\partial c+b
$$

Let us indicate how $b$ and $c$ can be found: Choose an ordering of the vertices of $P$, let $T_{0}$ be the corresponding pulling triangulation of $P$ (obtained from the trivial subdivision of $P$ by successively pulling the vertices), and let $z_{0} \in C_{n}(P, \partial P)$ be the corresponding cycle. Let $T_{1}$ be the triangulation of $P$ giving rise to $z_{1}$. The point is that there exists a triangulation $T$ of the product polytope $P \times[0,1]$ which restricts to $T_{0}$ on $P \times\{0\}$ and to $T_{1}$ on $P \times\{1\}$. (Note that this triangulation $T$ is easy to describe explicitly.) The corresponding affine chain $z_{T} \in C_{n+1}(P \times[0,1])$ projects to an affine chain $c_{1} \in C_{n+1}(P)$ via the natural projection $P \times[0,1] \rightarrow P$. Furthermore, let $b_{1} \in$ $C_{n}(\partial P)$ denote the image under the natural projection of the affine chain in $C_{n}(\partial P \times$ $[0,1]) \subset C_{n}(\partial(P \times[0,1]))$ corresponding to the restriction of the triangulation $T$ to the subset $\partial P \times[0,1]$ of the boundary of $P \times[0,1]$. By construction we have $z_{1}-z_{0}+b_{1}=\partial c_{1}$. Symmetrically, one gets $z_{2}-z_{0}+b_{2}=\partial c_{2}$, so that $z_{2}-z_{1}=$ $\partial\left(c_{2}-c_{1}\right)+\left(b_{1}-b_{2}\right)$.

Let $C^{q}(P)$ denote the algebraic dual of the space of chains $C_{q}(P)$. The relative cochain complex of $(P, \partial P)$ is given, in degree $q$, by

$$
C^{q}(P, \partial P)=\left\{f \in C^{q}(P) \mid f(c)=0 \text { for every } c \in C_{q}(\partial P)\right\} .
$$


The coboundary operator $\delta: C^{q}(P) \rightarrow C^{q+1}(P)$ clearly restricts to the relative cochain complex $C^{*}(P, \partial P)$, and the resulting cohomology group $H^{*}(P, \partial P)$ is the relative cohomology of $(P, \partial P)$. Observe that the evaluation of chains on cochains induces a well-defined pairing

$$
\langle., .\rangle: H^{q}(P, \partial P) \otimes H_{q}(P, \partial P) \longrightarrow \mathbb{R} \text {. }
$$

Finally, using some homological algebra, let us give another proof of the fact that two affine cycles $z_{1}, z_{2}$ arising from two triangulations of an $n$-dimensional connected polytope $P$ produce the same homology class in $H_{n}(P, \partial P)$ : By the Poincaré duality we know that $H_{n}(P, \partial P) \cong \mathbb{R}$, thus there exists $\lambda \in \mathbb{R}$ such that $\left[z_{1}\right]=\lambda\left[z_{2}\right]$. Define the cocycle $\operatorname{Vol}_{P} \in C^{n}(P, \partial P)$ as the Euclidean volume. Clearly, its evaluation on both $z_{1}$ and $z_{2}$ is equal to the volume $\operatorname{Vol}(P)$ of $P$, and hence

$$
\operatorname{Vol}(P)=\left\langle\left[\operatorname{Vol}_{P}\right],\left[z_{1}\right]\right\rangle=\left\langle\left[\operatorname{Vol}_{P}\right], \lambda\left[z_{2}\right]\right\rangle=\lambda \operatorname{Vol}(P) .
$$

It follows that $\lambda=1$, as claimed. The case where $P$ is not connected easily follows from the connected case.

\subsection{Combinatorial Volume}

We start with a trivial case: The cocycle $v:\left(S^{0}\right)^{2} \rightarrow \mathbb{R}$ determines the cocycle

$$
v \in C^{1}([0,1],\{0,1\}),
$$

which we still denote by $v$, defined as

$$
v(\sigma)=v\left(\sigma_{0}, \sigma_{1}\right)
$$

where $\sigma_{0}, \sigma_{1}$ are the (ordered) vertices of the affine simplex $\sigma$. Note that, obviously, $v$ is well defined, since it vanishes on 1-chains contained in the boundary. If $T$ is the only triangulation of the interval with all its vertices in the boundary, then the relative cycle $z_{T}$, which consists of one affine chain, satisfies $v\left(z_{T}\right)=1$. Clearly, $v$ is nothing else than the Euclidean volume.

Choose an embedding of the convex polygon $P(m)$ with $m$ vertices in the closed unit disk $\mathbb{D}^{2}$ in such a way that all the vertices $P(m)^{0}$ of $P(m)$ lie on the boundary $S^{1}$ of $\mathbb{D}^{2}$. The orientation cocycle determines the cocycle

$$
\text { Or } \in C^{2}(P(m), \partial P(m)),
$$

which we still denote by Or, defined as

$$
\operatorname{Or}(\sigma)=\operatorname{Or}\left(\sigma_{0}, \sigma_{1}, \sigma_{2}\right)
$$

where $\sigma_{0}, \sigma_{1}, \sigma_{2} \in P(m)^{0} \subset S^{1}$ are the (ordered) vertices of the affine simplex $\sigma$. Note that $\mathrm{Or}$ is well defined since if $\sigma$ is an affine simplex in $\partial P(m)$, then two of its vertices must be equal, so that $\operatorname{Or}(\sigma)=0$. If $T$ is a triangulation of $P(m)$ in $m-2$ simplices of dimension 2 with all its vertices in $P(m)^{0}$, then the relative cycle 
$z_{T}$ satisfies $\operatorname{Or}\left(z_{T}\right)=m-2$. Moreover, since the evaluation of Or on two cycles arising from two triangulations of $P(m)$ is constant and $\operatorname{Or}(\sigma)=0$ if and only if $\sigma \subset \partial P(m)$, it is immediate that any triangulation of $P(m)$ with all its vertices in the boundary must have precisely $m-2$ simplices of dimension 2 . Of course, taking $\mathbb{D}^{2}$ to be Klein's projective model for the hyperbolic 2-space, $\pi \cdot \operatorname{Or}(\sigma)$ is nothing else than the hyperbolic volume (or area) of $\sigma$.

The cocycles $\mathrm{Vol}_{3}$ and Or $\cup v:\left(S^{1} \times S^{0}\right)^{3} \rightarrow \mathbb{R}$ determine the cocycles

$$
\mathrm{Vol}_{3} \text { and } \mathrm{Or} \cup v \in C^{3}(P(m) \times[0,1], \partial(P(m) \times[0,1]))
$$

by

$$
\operatorname{Vol}_{3}(\sigma)=\operatorname{Vol}_{3}\left(\sigma_{0}, \sigma_{1}, \sigma_{2}, \sigma_{3}\right) \text { and } \operatorname{Or} \cup v(\sigma)=\operatorname{Or} \cup v\left(\sigma_{0}, \sigma_{1}, \sigma_{2}, \sigma_{3}\right) \text {, }
$$

where $\sigma_{0}, \sigma_{1}, \sigma_{2}, \sigma_{3}$ are the (ordered) vertices of the affine simplex $\sigma$. To check that those two cocycles are well defined, we need to verify that they vanish on affine chains contained in $\partial(P(m) \times[0,1])$. Such chains are linear combinations of two types of affine simplices: those contained in $P(m) \times\{*\}$ and those contained in $\tau \times$ $[0,1]$, where $\tau$ is an exterior edge of $P(m)$. In the former case, all the $\nu$-factors appearing in the definitions of $\mathrm{Vol}_{3}$ and $\mathrm{Or} \cup v$ vanish, and in the latter case, all the Or-factors do, so in either cases, the two cocycles vanish. For the same reason, the cochain $b_{3}$ (whose coboundary is the difference between Or $\cup v$ and $V_{0} l_{3}$ ) also is a cochain in $C^{2}(P(m) \times[0,1], \partial(P(m) \times[0,1]))$, so that

$$
\left[\mathrm{Vol}_{3}\right]=[\mathrm{Or} \cup v] \in H^{3}(P(m) \times[0,1], \partial(P(m) \times[0,1])) .
$$

Proposition 3 Let $T$ be any triangulation of the prism $P(m) \times[0,1]$ with all its vertices in $(P(m) \times[0,1])^{0}$. Then

$$
\operatorname{Vol}_{3}\left(z_{T}\right)=m-2 .
$$

Proof If $z$ and $z^{\prime}$ are two affine chains on $P(m) \times[0,1]$ coming from two triangulations $T$ and $T^{\prime}$ of the prism, then they determine the same homology class $[z]$ in $H_{3}(P(m) \times[0,1], \partial(P(m) \times[0,1]))$, and

$$
\operatorname{Vol}_{3}(z)=\left\langle\left[\operatorname{Vol}_{3}\right],[z]\right\rangle=\operatorname{Vol}_{3}\left(z^{\prime}\right) .
$$

Let now $T_{0}$ be a triangulation of $P(m)$ in $m-2$ simplices of dimension 2 . After choosing a numbering of the vertices of $P(m)$ and those of $[0,1]$, we get a canonical triangulation $T$ of $P(m) \times[0,1]$ (in 3(m-2) simplices of dimension 3). Denoting by $z_{0}$ and $z_{T}$ the affine cycles arising from the triangulations of $T_{0}$ and $T$ respectively, we have

$$
\operatorname{Vol}_{3}\left(z_{T}\right)=\left\langle\left[\operatorname{Vol}_{3}\right],\left[z_{T}\right]\right\rangle=(\operatorname{Or} \cup v)\left(z_{T}\right)=\operatorname{Or}\left(z_{0}\right) \cdot v([0,1])=m-2,
$$

which finishes the proof of the proposition.

The cocycles $\mathrm{Vol}_{4}$ and Or $\cup$ Or $:\left(S^{1} \times S^{1}\right)^{5} \rightarrow \mathbb{R}$ determine the cocycles

$$
\mathrm{Vol}_{4} \text { and } \text { Or } \cup \text { Or } \in C^{5}(P(m) \times P(n), \partial(P(m) \times P(n)))
$$


by

$$
\operatorname{Vol}_{4}(\sigma)=\operatorname{Vol}_{4}\left(\sigma_{0}, \ldots, \sigma_{4}\right) \quad \text { and } \quad \operatorname{Or} \cup \operatorname{Or}(\sigma)=\operatorname{Or} \cup \operatorname{Or}\left(\sigma_{0}, \ldots, \sigma_{4}\right) \text {, }
$$

where $\sigma_{0}, \ldots, \sigma_{4}$ are the (ordered) vertices of the affine simplex $\sigma$. To check that those two cocycles are well defined, we need to verify that they vanish on affine chains contained in $\partial(P(m) \times P(n))$. Such chains are linear combinations of two types of affine simplices: those contained in $P(m) \times \tau$ and those contained in $\tau \times P(n)$, where $\tau$ is an exterior edge of $P(n)$ or $P(m)$, respectively. In the former case, all the Or-factors in the second factor appearing in the definitions of $\mathrm{Vol}_{4}$ and Or $\cup$ Or vanish, and in the latter case, all the Or-factors in the first factor do, so in either cases, the two cocycles vanish. For the same reason, the cochain $b_{4}$ (whose coboundary is the difference between Or $\cup$ Or and $\mathrm{Vol}_{4}$ ) also is a cochain in $C^{3}(P(m) \times P(n), \partial(P(m) \times P(n)))$, so that

$$
\left[\mathrm{Vol}_{4}\right]=[\mathrm{Or} \cup \mathrm{Or}] \in H^{4}(P(m) \times P(n), \partial(P(m) \times P(n))) .
$$

Proposition 4 Let $T$ be any triangulation of the product $P(m) \times P(n)$. Then

$$
\operatorname{Vol}_{4}\left(z_{T}\right)=(m-2)(n-2) .
$$

Proof The proof that $\operatorname{Vol}_{4}\left(z_{T}\right)$ is independent of the triangulation $T$ is identical to the proof of the analogous statement in Proposition 3.

Let now $T_{m}$ and $T_{n}$ be triangulations of $P(m)$, respectively $P(n)$, in $m-2$, resp. $n-2$, simplices of dimension 2 . After choosing a numbering of the vertices of $P(m)$ and $P(n)$, we get a canonical triangulation $T$ of $P(m) \times P(n)($ in $6(m-2)(n-2)$ simplices of dimension 4). Denoting by $z_{m}, z_{n}$, and $z_{T}$ the affine cycles arising from the triangulations of $T_{m}, T_{n}$, and $T$, respectively, we have

$$
\operatorname{Vol}_{4}\left(z_{T}\right)=\left\langle\left[\operatorname{Vol}_{4}\right],\left[z_{T}\right]\right\rangle=(\text { Or } \cup \text { Or })\left(z_{T}\right)=\operatorname{Or}\left(z_{m}\right) \cdot \operatorname{Or}\left(z_{n}\right)=(m-2)(n-2),
$$

which finishes the proof of the proposition.

We will call the cocycles $v, \mathrm{Or}, \mathrm{Vol}_{3}$, and $\mathrm{Vol}_{4}$ combinatorial volume cocycles. In fact, for products of intervals, triangles, and squares, we obtain the Euclidean volume cocycle (up to a constant coming from that our triangles have area equal to 1 instead of $1 / 2$ ). In particular, our proof of the minimality of triangulations of the 3-cube and 4 -cube with 5 , respectively 16 , top dimensional simplices is nothing else than a classical (Euclidean) volume argument.

\subsection{Values of $\mathrm{Vol}_{3}$}

In order to examine the various possible triangulations of the prism $P(m) \times[0,1]$, we need to study the possible values of the combinatorial volume cocycle $\mathrm{Vol}_{3}$. Expanding the sum in the defining expression for $\mathrm{Vol}_{3}$, we get 


$$
\begin{aligned}
\operatorname{Vol}_{3}\left(\left(x_{0}, y_{0}\right), \ldots,\left(x_{3}, y_{3}\right)\right)= & \frac{1}{12}\left[\operatorname{Or}\left(x_{0}, x_{1}, x_{2}\right)\left(v\left(y_{2}, y_{3}\right)+v\left(y_{1}, y_{3}\right)+v\left(y_{0}, y_{3}\right)\right)\right. \\
& +\operatorname{Or}\left(x_{0}, x_{1}, x_{3}\right)\left(v\left(y_{2}, y_{3}\right)-v\left(y_{1}, y_{2}\right)-v\left(y_{0}, y_{2}\right)\right) \\
& +\operatorname{Or}\left(x_{0}, x_{2}, x_{3}\right)\left(-v\left(y_{1}, y_{3}\right)-v\left(y_{1}, y_{2}\right)+v\left(y_{0}, y_{1}\right)\right) \\
& \left.+\operatorname{Or}\left(x_{1}, x_{2}, x_{3}\right)\left(v\left(y_{0}, y_{3}\right)+v\left(y_{0}, y_{2}\right)+v\left(y_{0}, y_{1}\right)\right)\right]
\end{aligned}
$$

for every 4-tuple $\left(\left(x_{0}, y_{0}\right), \ldots,\left(x_{3}, y_{3}\right)\right)$ in $\left(S^{1} \times S^{0}\right)^{4}$. If $y_{0}=y_{1}=y_{2}=y_{3}$, then $v\left(y_{i}, y_{j}\right)=0$ for every $i, j$, so that the volume cocycle $\mathrm{Vol}_{3}$ clearly vanishes. If the $y_{i}$ 's are not all equal, then the cardinality of the set $\left\{y_{0}, \ldots, y_{3}\right\}$ has to be equal to 2 , in which case, upon permuting the variables and the points 0 and 1 of $S^{0}$, we distinguish two cases:

- $y_{0}=y_{1}=y_{2}=0$ and $y_{3}=1$ : Using the cocycle relation $\delta \operatorname{Or}\left(x_{0}, x_{1}, x_{2}, x_{3}\right)=0$, we compute that $\operatorname{Vol}_{3}\left(\left(x_{0}, y_{0}\right), \ldots,\left(x_{3}, y_{3}\right)\right)$ is equal to

$$
\begin{aligned}
& \frac{1}{12}\left[3 \operatorname{Or}\left(x_{0}, x_{1}, x_{2}\right)+\operatorname{Or}\left(x_{0}, x_{1}, x_{3}\right)-\operatorname{Or}\left(x_{0}, x_{2}, x_{3}\right)+\operatorname{Or}\left(x_{1}, x_{2}, x_{3}\right)\right] \\
& \quad=\frac{1}{3} \operatorname{Or}\left(x_{0}, x_{1}, x_{2}\right) \in\left\{-\frac{1}{3}, 0, \frac{1}{3}\right\} .
\end{aligned}
$$

- $y_{0}=y_{1}=0$ and $y_{2}=y_{3}=+1$ : Let first the points $x_{0}, \ldots, x_{3} \in S^{1}$ be arbitrary. We have

$$
\begin{aligned}
\operatorname{Vol}_{3}\left(\left(x_{0}, y_{0}\right), \ldots,\left(x_{3}, y_{3}\right)\right) \\
=\frac{1}{6}\left[\operatorname{Or}\left(x_{0}, x_{1}, x_{2}\right)-\operatorname{Or}\left(x_{0}, x_{1}, x_{3}\right)-\operatorname{Or}\left(x_{0}, x_{2}, x_{3}\right)+\operatorname{Or}\left(x_{1}, x_{2}, x_{3}\right)\right] \\
=\frac{1}{3}\left[\operatorname{Or}\left(x_{0}, x_{1}, x_{2}\right)-\operatorname{Or}\left(x_{0}, x_{1}, x_{3}\right)\right]
\end{aligned}
$$

where we have used the cocycle relation $\delta \operatorname{Or}\left(x_{0}, x_{1}, x_{2}, x_{3}\right)=0$ for the last equality. Let $D_{01}$ and $D_{23}$ denote the segments between $x_{0}, x_{1}$ and $x_{2}, x_{3}$, respectively. There are now four possibilities for the intersection of those segments:

- $D_{01}=D_{23}$ : We have either $\left(x_{0}=x_{2}\right.$ and $\left.x_{1}=x_{3}\right)$ or $\left(x_{0}=x_{3}\right.$ and $\left.x_{1}=x_{2}\right)$. In either cases, $\mathrm{Vol}_{3}$ vanishes.

- $D_{01} \cap D_{23}$ intersect in one boundary point in $S^{1}$ : In view of the obvious symmetries, we can suppose that $x_{1}=x_{3}$, and then

$$
\operatorname{Vol}_{3}\left(\left(x_{0}, y_{0}\right), \ldots,\left(x_{3}, y_{3}\right)\right)=\frac{1}{3} \operatorname{Or}\left(x_{0}, x_{1}, x_{2}\right) \text {. }
$$

- The four points $x_{0}, x_{1}, x_{2}, x_{3}$ are all different. In this case, we have $\operatorname{Or}\left(x_{0}, x_{1}, x_{2}\right)= \pm \operatorname{Or}\left(x_{0}, x_{1}, x_{3}\right)$ with the minus sign happening if and only if $D_{01}$ and $D_{23}$ intersect in one interior point, in which case the volume is $(2 / 3) \operatorname{Or}\left(x_{0}, x_{1}, x_{2}\right)$ with the plus sign happening if and only if the intersection $D_{01} \cap D_{23}$ is empty, in which case the volume is zero. 


\subsection{Values of $\mathrm{Vol}_{4}$}

As we want to estimate the value of $\mathrm{Vol}_{4}$ on different configurations of points, it would be convenient to have a simpler expression for it. We will now show that in the defining expression for $\mathrm{Vol}_{4}$, it is enough to average over those permutations mapping 2 to 0 , so that we will obtain the expression $(\diamond)$ below. Fix $\left(z_{0}, \ldots, z_{4}\right)$ in $\left(S^{1} \times S^{1}\right)^{5}$. We start by showing that

$$
\Phi(i):=\frac{1}{4} \sum_{\substack{\sigma \in \operatorname{Sym}(5) \\ \sigma(2)=i}} \operatorname{sign}(\sigma)(\mathrm{Or} \cup \text { Or })\left(z_{\sigma(0)}, z_{\sigma(1)}, z_{i}, z_{\sigma(3)}, z_{\sigma(4)}\right)
$$

is independent of $i$. By definition, $\Phi(0)$ is equal to

$$
\begin{aligned}
& \operatorname{Or}\left(x_{0}, x_{1}, x_{2}\right) \operatorname{Or}\left(y_{0}, y_{3}, y_{4}\right)+\operatorname{Or}\left(x_{0}, x_{3}, x_{4}\right) \operatorname{Or}\left(y_{0}, y_{1}, y_{2}\right) \\
& \quad-\operatorname{Or}\left(x_{0}, x_{1}, x_{3}\right) \operatorname{Or}\left(y_{0}, y_{2}, y_{4}\right)-\operatorname{Or}\left(x_{0}, x_{2}, x_{4}\right) \operatorname{Or}\left(y_{0}, y_{1}, y_{3}\right) \\
& +\operatorname{Or}\left(x_{0}, x_{1}, x_{4}\right) \operatorname{Or}\left(y_{0}, y_{2}, y_{3}\right)+\operatorname{Or}\left(x_{0}, x_{2}, x_{3}\right) \operatorname{Or}\left(y_{0}, y_{1}, y_{4}\right),
\end{aligned}
$$

where we have used, on the first factors, the fact that Or is alternating. From the cocycle relation for Or, we have, for $i, j$ in $\{2,3,4\}$,

$$
\operatorname{Or}\left(x_{0}, x_{i}, x_{j}\right)=\operatorname{Or}\left(x_{1}, x_{i}, x_{j}\right)+\operatorname{Or}\left(x_{0}, x_{1}, x_{j}\right)-\operatorname{Or}\left(x_{0}, x_{1}, x_{i}\right)
$$

and an analogous formula for the $y_{i}$ 's. The previous expression thus becomes

$$
\begin{aligned}
\operatorname{Or} & \left(x_{0}, x_{1}, x_{2}\right)\left(\operatorname{Or}\left(y_{1}, y_{3}, y_{4}\right)+\operatorname{Or}\left(y_{0}, y_{1}, y_{4}\right)-\operatorname{Or}\left(y_{0}, y_{1}, y_{3}\right)\right) \\
& +\left(\operatorname{Or}\left(x_{1}, x_{3}, x_{4}\right)+\operatorname{Or}\left(x_{0}, x_{1}, x_{4}\right)-\operatorname{Or}\left(x_{0}, x_{1}, x_{3}\right)\right) \operatorname{Or}\left(y_{0}, y_{1}, y_{2}\right) \\
& -\operatorname{Or}\left(x_{0}, x_{1}, x_{3}\right)\left(\operatorname{Or}\left(y_{0}, y_{2}, y_{4}\right)+\operatorname{Or}\left(y_{0}, y_{1}, y_{4}\right)-\operatorname{Or}\left(y_{0}, y_{1}, y_{2}\right)\right) \\
& -\left(\operatorname{Or}\left(x_{1}, x_{2}, x_{4}\right)+\operatorname{Or}\left(x_{0}, x_{1}, x_{4}\right)-\operatorname{Or}\left(x_{0}, x_{1}, x_{2}\right)\right) \operatorname{Or}\left(y_{0}, y_{1}, y_{3}\right) \\
& +\operatorname{Or}\left(x_{0}, x_{1}, x_{4}\right)\left(\operatorname{Or}\left(y_{0}, y_{2}, y_{3}\right)+\operatorname{Or}\left(y_{0}, y_{1}, y_{4}\right)-\operatorname{Or}\left(y_{0}, y_{1}, y_{2}\right)\right) \\
& +\left(\operatorname{Or}\left(x_{1}, x_{2}, x_{3}\right)+\operatorname{Or}\left(x_{0}, x_{1}, x_{3}\right)-\operatorname{Or}\left(x_{0}, x_{1}, x_{2}\right)\right) \operatorname{Or}\left(y_{0}, y_{1}, y_{4}\right) .
\end{aligned}
$$

Now note that all the terms of the form $\operatorname{Or}\left(x_{0}, x_{1}, x_{i}\right) \operatorname{Or}\left(y_{0}, y_{1}, y_{j}\right)$ for $i, j$ in $\{2,3,4\}$ cancel out two by two, and what we are left with is precisely $\Phi(1)$. We have thus proven that $\Phi(0)=\Phi(1)$. Applying the cyclic permutation $(0,1, \ldots, 4)$ and its powers to the indices in the proof of the latter equality, it is immediate that $\Phi(1)=\Phi(2)=\Phi(3)=\Phi(4)$. We thus have $\operatorname{Vol}_{4}\left(\left(x_{0}, y_{0}\right), \ldots,\left(x_{4}, y_{4}\right)\right)=$ $(1 / 30)(\Phi(0)+\Phi(1)+\Phi(2)+\Phi(3)+\Phi(4))=(1 / 6) \Phi(0)$, and we have hence proven the following equation:

$$
\begin{aligned}
\operatorname{Vol}_{4} & \left(\left(x_{0}, y_{0}\right), \ldots,\left(x_{4}, y_{4}\right)\right) \\
= & \frac{1}{6}\left(\operatorname{Or}\left(x_{0}, x_{1}, x_{2}\right) \operatorname{Or}\left(y_{0}, y_{3}, y_{4}\right)+\operatorname{Or}\left(x_{0}, x_{3}, x_{4}\right) \operatorname{Or}\left(y_{0}, y_{1}, y_{2}\right)\right. \\
& \quad-\operatorname{Or}\left(x_{0}, x_{1}, x_{3}\right) \operatorname{Or}\left(y_{0}, y_{2}, y_{4}\right)-\operatorname{Or}\left(x_{0}, x_{2}, x_{4}\right) \operatorname{Or}\left(y_{0}, y_{1}, y_{3}\right) \\
& \left.+\operatorname{Or}\left(x_{0}, x_{1}, x_{4}\right) \operatorname{Or}\left(y_{0}, y_{2}, y_{3}\right)+\operatorname{Or}\left(x_{0}, x_{2}, x_{3}\right) \operatorname{Or}\left(y_{0}, y_{1}, y_{4}\right)\right) .
\end{aligned}
$$


Lemma 5 If three among the $x_{i}$ 's are equal or if three among the $y_{i}$ 's are equal, then

$$
\left|\operatorname{Vol}_{4}\left(\left(x_{0}, y_{0}\right), \ldots,\left(x_{4}, y_{4}\right)\right)\right| \leq \frac{1}{6}
$$

Proof As $\mathrm{Vol}_{4}$ is alternating and symmetric in the first and second factor, we can without loss of generality assume that $x_{0}=x_{1}=x_{2}$. But then, the expression $(\diamond)$ for $\mathrm{Vol}_{4}$ reduces to

$$
\frac{1}{6} \operatorname{Or}\left(x_{0}, x_{3}, x_{4}\right) \operatorname{Or}\left(y_{0}, y_{1}, y_{2}\right) \in\left\{-\frac{1}{6}, 0, \frac{1}{6}\right\} .
$$

Lemma 6 If there exists $i_{1}, i_{2}$ and $j_{1}, j_{2}$ all distinct such that either $x_{i_{1}}=x_{i_{2}}$ and $x_{j_{1}}=x_{j_{2}}$, or $y_{i_{1}}=y_{i_{2}}$ and $y_{j_{1}}=y_{j_{2}}$, then

$$
\left|\operatorname{Vol}_{4}\left(\left(x_{0}, y_{0}\right), \ldots,\left(x_{4}, y_{4}\right)\right)\right| \leq \frac{1}{3} .
$$

Proof As $\mathrm{Vol}_{4}$ is alternating and symmetric in the first and second factor, we can without loss of generality assume that $x_{0}=x_{1}$ and $x_{2}=x_{3}$. The expression $(\diamond)$ for $\mathrm{Vol}_{4}$ now reduces to

$$
\frac{1}{6}\left(\operatorname{Or}\left(x_{0}, x_{3}, x_{4}\right) \operatorname{Or}\left(y_{0}, y_{1}, y_{2}\right)-\operatorname{Or}\left(x_{0}, x_{2}, x_{4}\right) \operatorname{Or}\left(y_{0}, y_{1}, y_{3}\right)\right) .
$$

Lemma 7 If there exist $i \neq j$ such that $x_{i}=x_{j}$ or $y_{i}=y_{j}$, then

$$
\left|\operatorname{Vol}_{4}\left(\left(x_{0}, y_{0}\right), \ldots,\left(x_{4}, y_{4}\right)\right)\right| \leq \frac{1}{2}
$$

Proof $\mathrm{As}_{\mathrm{Vol}_{4}}$ is alternating, we can without loss of generality assume that $x_{0}=x_{1}$, so that the expression $(\diamond)$ for $\mathrm{Vol}_{4}$ reduces to

$$
\begin{aligned}
& \frac{1}{6}\left[\operatorname{Or}\left(x_{0}, x_{3}, x_{4}\right) \operatorname{Or}\left(y_{0}, y_{1}, y_{2}\right)-\operatorname{Or}\left(x_{0}, x_{2}, x_{4}\right) \operatorname{Or}\left(y_{0}, y_{1}, y_{3}\right)\right. \\
& \left.\quad+\operatorname{Or}\left(x_{0}, x_{2}, x_{3}\right) \operatorname{Or}\left(y_{0}, y_{1}, y_{4}\right)\right] .
\end{aligned}
$$

Lemma 8 Let $\left(\left(x_{0}, y_{0}\right), \ldots,\left(x_{4}, y_{4}\right)\right)$ be an arbitrary 5-tuple in $\left(S^{1} \times S^{1}\right)^{5}$. Then

$$
\left|\operatorname{Vol}_{4}\left(\left(x_{0}, y_{0}\right), \ldots,\left(x_{4}, y_{4}\right)\right)\right| \leq \frac{2}{3}
$$

Proof First note that if either all the $x_{i}$ 's are not distinct or all the $y_{i}$ 's are not distinct, then by Lemma 7, the evaluation of $\mathrm{Vol}_{4}$ is at most $1 / 2$. Thus we can assume that this is not the case. Since $\mathrm{Vol}_{4}$ is alternating, we can furthermore assume that the 
$x_{i}$ 's are positively cyclically ordered. In particular, $\operatorname{Or}\left(x_{i}, x_{j}, x_{k}\right)=+1$, whenever $0 \leq i<j<k \leq 4$. The expression $(\diamond)$ for $\mathrm{Vol}_{4}$ now becomes

$$
\begin{aligned}
& \frac{1}{6}\left[\operatorname{Or}\left(y_{0}, y_{1}, y_{2}\right)-\operatorname{Or}\left(y_{0}, y_{1}, y_{3}\right)+\operatorname{Or}\left(y_{0}, y_{1}, y_{4}\right)\right. \\
& \left.\quad+\operatorname{Or}\left(y_{0}, y_{3}, y_{4}\right)-\operatorname{Or}\left(y_{0}, y_{2}, y_{4}\right)+\operatorname{Or}\left(y_{0}, y_{2}, y_{3}\right)\right] .
\end{aligned}
$$

Using the cocycle relation

$$
\delta \operatorname{Or}\left(y_{0}, y_{2}, y_{3}, y_{4}\right)=0 \text {, }
$$

we see that the above expression can be rewritten as

$$
\frac{1}{6}\left(\operatorname{Or}\left(y_{0}, y_{1}, y_{2}\right)-\operatorname{Or}\left(y_{0}, y_{1}, y_{3}\right)+\operatorname{Or}\left(y_{0}, y_{1}, y_{4}\right)+\operatorname{Or}\left(y_{2}, y_{3}, y_{4}\right)\right) \leq 2 / 3 \text {, }
$$

which proves the lemma.

\section{Minimal Triangulations of the Prism $P(m) \times[0,1]$}

The following result was first proven in [3].

Theorem 9 The minimal number of 3-simplices in a triangulation of the prism $P(m) \times[0,1]$ is equal to

$$
\begin{cases}\frac{5}{2} m-5 & \text { if } m \text { is even, } \\ \frac{5}{2} m-\frac{9}{2} & \text { if } m \text { is odd }\end{cases}
$$

Proof Let $T$ be a fixed triangulation of $P(m) \times[0,1]$. By restriction, the triangulation $T$ gives triangulations of each of the $m$-gons $P(m) \times\{0\}$ and $P(m) \times\{1\}$ in $m-2$ simplices of dimension 2. Each of those 2-simplices is a 2-face of one and only one 3 -simplex of $T$. We call such a 3-simplex a simplex of type $\operatorname{Tr}$ (for triangle in an $m$-gon). There are precisely

$$
2(m-2)
$$

simplices of type Tr. In view of the computations of Sect. 2.4, a simplex $\sigma$ of type $\operatorname{Tr}$ has the combinatorial volume

$$
\left|\operatorname{Vol}_{3}(\sigma)\right|=\frac{1}{3}
$$

All the remaining simplices of $T$ have two vertices in $P(m) \times\{0\}$ and two in $P(m) \times\{1\}$. We will say that such an ordered simplex $\sigma$ is of type $A$ if $\operatorname{Vol}_{3}(\sigma)=$ $+2 / 3$ and of type $B$ if $\operatorname{Vol}_{3}(\sigma)=+1 / 3$. Denote by $a$, respectively $b$, the number of simplices of type $A$, resp. $B$, in $T$. 
Let $z_{T}$ be the affine cycle arising from the triangulation $T$. It follows from Proposition 3 that $\operatorname{Vol}_{3}\left(z_{T}\right)=m-2$, and hence

$$
m-2 \leq \frac{1}{3} \cdot 2(m-2)+\frac{2}{3} a+\frac{1}{3} b,
$$

where the last inequality comes from that we have only counted the contribution to the combinatorial volume coming from simplices of type $\operatorname{Tr}, A$ and $B$. There may be more simplices, but as computed in Sect. 2.4, they have $\operatorname{Vol}_{3}(\sigma) \leq 0$.

Since $b$ is positive, we get from the previous inequality that

$$
a+b \geq a+\frac{1}{2} b \geq \frac{1}{2}(m-2) .
$$

The number of 3-dimensional simplices of $T$ is hence greater or equal to

$$
2(m-2)+a+b \geq \frac{5}{2}(m-2) .
$$

Rounding up when $m$ is an odd integer gives the claimed lower bound.

For the equality, note that there exists a triangulation of $P(3) \times[0,1]$ in 3 simplices ( 2 of type $\operatorname{Tr}$ and 1 of type $B$ ). There exists a triangulation of $P(4) \times[0,1]$ in 5 simplices (4 of type Tr and 1 of type $A$ ). Decompose $P(2 n) \times[0,1]$ in $(2 n-2)$ cubes $P(4) \times[0,1]$ and triangulate each of those minimally in 5 simplices. Decompose $P(2 n+1) \times[0,1]$ in $(2 n-2)$ cubes $P(4) \times[0,1]$ and one prism $P(3) \times[0,1]$ and triangulate each of those minimally in 5 , respectively 3 , simplices.

\section{The Boundary Simplices of a Triangulation of $P(m) \times P(n)$}

Theorem 10 Let $T$ be any triangulation of $P(m) \times P(n)$. The number of 4-simplices of $T$ with a 3 -face in $\partial(P(m) \times P(n))$ is greater or equal to

$$
\frac{5}{2} m n-3(m+n)
$$

Observe that this already gives a lower bound for the minimal number $T(m, n)$ of 4-simplices in a triangulation of $P(m) \times P(n)$ which improves the lower bound of $2 m n-(8 / 3)(m+n)$ computed in [1]. Using our combinatorial volume cocycle $\mathrm{Vol}_{4}$, we will further improve this lower bound in the next section.

Proof Let $T$ be a fixed triangulation of $P(m) \times P(n)$. Its restriction to every prism $P(m) \times \tau$ or $\tau \times P(n)$, where $\tau$ is a boundary edge of $P(n)$ or $P(m)$, has 3-simplices of (at least) three types, according to the terminology introduced in the proof of Theorem 9, type $\operatorname{Tr}$, type $A$, and type $B$. We will say that a 4-simplex of the triangulation $T$ is of type $A_{m}$ or $B_{m}$ (respectively $A_{n}$ or $B_{n}$ ) if one of its 3-face is contained in some prism $P(m) \times \tau$ (resp. $\tau \times P(n))$ and of type $A$, respectively $B$. As for the 4-simplices with a 3-face of type Tr, we distinguish two cases, according to the number of 3-face of type $\operatorname{Tr}$ in $P(m) \times \tau$ (resp. $\tau \times P(n)$ ): A 4-simplex is of type $\operatorname{Tr}_{m, 1}$ 
(respectively $\operatorname{Tr}_{n, 1}$ ) if it has precisely one 3-faces of type $\operatorname{Tr}$ in a prism of the form $P(m) \times \tau$ (resp. $\tau \times P(n)$ ) and of type $\operatorname{Tr}_{m, 2}$ (respectively $\operatorname{Tr}_{n, 2}$ ) if it has precisely two 3-faces of type Tr in prisms of the form $P(m) \times \tau$ (resp. $\tau \times P(n)$ ). Note that a 4-simplex cannot have three faces of type Tr in prisms of the form $P(m) \times \tau$ (resp. $\tau \times P(n))$.

The different types are not necessarily exclusive. It is for example possible for a 4-simplex to be both of type $\operatorname{Tr}_{m, 2}$ and $\operatorname{Tr}_{n, 2}$, as one can see in the 4-cube for the simplex obtained by chopping off a corner. In this case, we will say that the 4simplex is of type $\operatorname{Tr}_{m, 2} \cap \operatorname{Tr}_{n, 2}$. More generally, if a 4-simplex is both of type $X$ and $Y$, we will say that it is of type $X \cap Y$. In the two next claims, we will study the possible intersections of different types. Before we do that, let us introduce some more notation.

For $k=m$ or $n$, define $t_{k, 1}, t_{k, 2}, a_{k}, b_{k}$ to be the number of 4-simplices in the triangulation $T$ of type $\operatorname{Tr}_{k, 1}, \operatorname{Tr}_{k, 2}, A_{k}$, or $B_{k}$ respectively. Also, for $x=t_{m, 1}, t_{m, 2}, a_{m}, b_{m}$ and $y=t_{n, 1}, t_{n, 2}, a_{n}, b_{n}$, we let $x \cap y$ be the number of simplices of the corresponding types.

Claim 11 No 4-simplex can belong to more than one of the types $\operatorname{Tr}_{m, 1}, \operatorname{Tr}_{m, 2}, A_{m}$, and $B_{m}$, or to more than one of $\operatorname{Tr}_{n, 1}, \operatorname{Tr}_{n, 2}, A_{n}$, and $B_{n}$.

Proof By symmetry, it is enough to prove the first assertion. Let $\sigma$ be a 4-simplex of $T$ with a 3 -face in a given prism $P(m) \times \tau$. Thus, four of the vertices, say $\sigma_{1}, \sigma_{2}, \sigma_{3}, \sigma_{4}$ of $\sigma$ are vertices of $P(m) \times \tau$. The fifth vertex $\sigma_{0}$ of $\sigma$ cannot also belong to $P(m) \times \tau$ (otherwise the 4-dimensional simplex $\sigma$ would be contained in the 3-dimensional prism $P(m) \times \tau)$. Suppose that another 3-face of $\sigma$, say the one generated by $\sigma_{0}, \sigma_{1}, \sigma_{2}, \sigma_{3}$, belongs to another prism $P(m) \times \tau^{\prime}$. Necessarily, $\tau$ and $\tau^{\prime}$ have a vertex $y$ in common, and

$$
\sigma_{1}, \sigma_{2}, \sigma_{3} \in P(m) \times\{y\} .
$$

Thus, if two 3-faces of $\sigma$ belong to prisms of the form $P(m) \times \tau$, then $\sigma$ is of type $\operatorname{Tr}_{m, 2}$. Observe furthermore that no other 3-face of $\sigma$ can belong to a prism of the form $P(m) \times \tau$.

As a consequence, we see that the intersection of any three different types has to be empty. Furthermore, it immediately follows from Claim 11 that for any of the types $\operatorname{Tr}_{m, 1}, \operatorname{Tr}_{m, 2}, A_{m}$, or $B_{m}$, its intersections with the types $\operatorname{Tr}_{n, 1}, \operatorname{Tr}_{n, 2}, A_{n}$, and $B_{n}$ are disjoint. Thus, as $\operatorname{Tr}_{m, 1} \cap \operatorname{Tr}_{n, 1}, \operatorname{Tr}_{m, 1} \cap \operatorname{Tr}_{n, 2}$. and $\operatorname{Tr}_{m, 1} \cap B_{n}$ are disjoint subsets of $\operatorname{Tr}_{m, 1}$, we obtain the inequality

$$
t_{m, 1} \geq t_{m, 1} \cap t_{n, 1}+t_{m, 1} \cap t_{n, 2}+t_{m, 1} \cap b_{n} .
$$

The inequalities

$$
\begin{gathered}
t_{n, 1} \geq t_{m, 1} \cap t_{n, 1}+t_{m, 2} \cap t_{n, 1}+b_{m} \cap t_{n, 1}, \\
b_{m} \geq b_{m} \cap t_{n, 1}+b_{m} \cap t_{n, 2}+b_{m} \cap b_{n},
\end{gathered}
$$




$$
b_{n} \geq t_{m, 1} \cap b_{n}+t_{m, 2} \cap b_{n}+b_{m} \cap b_{n}
$$

are obtained analogously.

Claim 12 A 4-simplex of type $A_{m}$ or $A_{n}$ cannot be of a second type.

Proof Let $\sigma$ be a 4-simplex in the triangulation $T$. Let $p_{1}$ and $p_{2}$ denote the projections on the first, respectively second, factor of $P(m) \times P(n)$. Denote by $n_{1}(\sigma)$, respectively $n_{2}(\sigma)$, the cardinality of the image under $p_{1}$, resp. $p_{2}$, of the vertices of $\sigma$. If $\sigma$ is a simplex of type $A_{n}$, then $n_{1}(\sigma) \leq 3$ and $n_{2}(\sigma) \geq 4$. But if $\sigma$ is of one of the types $\operatorname{Tr}_{m, 1}, \operatorname{Tr}_{m, 2}, A_{m}$, and $B_{m}$, then $n_{2}(\sigma) \leq 3$, which is not possible. By symmetry, the assertion for $A_{m}$ is also proven.

Claim $132 a_{m}+b_{m} \geq n(m-2)$ and $2 a_{n}+b_{n} \geq m(n-2)$.

Proof By symmetry, it is enough to prove the first inequality of the claim. As in the proof of Theorem 9, in each prism $P(m) \times \tau$, the number $a$ of simplices of type $A$ and the number $b$ of simplices of type $B$ satisfy the inequality

$$
2 a+b \geq m-2 .
$$

As there are $n$ such prisms, and each 3-simplex of type $A$ or $B$ in $P(m) \times \tau$ belongs to one and only one 4-simplex of type $A_{m}$ or $B_{m}$, the claim follows.

Claim $14 t_{m, 1}+2 t_{m, 2}=2 n(m-2)$ and $t_{n, 1}+2 t_{n, 2}=2 m(n-2)$.

Proof By symmetry, it is enough to prove the first equality of the claim. Recall from the proof of Theorem 9 that in the restriction of the triangulation $T$ to any prism $P(m) \times \tau$, there are exactly $2(m-2)$ simplices of type Tr. As there are $n$ prisms of the form $P(m) \times \tau$ in $P(m) \times P(n)$, we have a total of

$$
2 n(m-2)
$$

3-simplices of type $\mathrm{Tr}$ in such prisms. (We do not count the simplices in prisms of the form $\tau \times P(n)$.)

By definition, a simplex of type $\operatorname{Tr}_{m, 1}$, respectively $\operatorname{Tr}_{m, 2}$, has precisely one, resp. two, 3-simplices as above. Since a 3-simplex in the boundary $\partial(P(m) \times P(n))$ belongs to one and only one 4-simplex of the triangulation $T$, it follows that

$$
t_{m, 1}+2 t_{m, 2}=2 n(m-2),
$$

as claimed.

Claim $154 t_{m, 2} \cap t_{n, 2}+2 t_{m, 1} \cap t_{n, 2}+2 t_{m, 2} \cap t_{n, 1}+2 t_{m, 2} \cap b_{n}+2 b_{m} \cap t_{n, 2} \leq 2 m n$.

Proof We count the number of triangles (i.e. 2-simplices) in the restriction of the triangulation $T$ to the union of the squares of the form $\tau_{m} \times \tau_{n}$, where $\tau_{m}$ and $\tau_{n}$ are 
boundary edges of $P(m)$ and $P(n)$, respectively. Clearly, each square is triangulated in two triangles, and there are $m n$ squares and hence a total of $2 m n$ triangles of the above form.

In a simplex of type $\operatorname{Tr}_{m, 2} \cap \operatorname{Tr}_{n, 2}$, there exist precisely 4 triangles of the above form: A simplex of type $\operatorname{Tr}_{m, 2}$ necessarily has three vertices in $P(m) \times\{y\}$ for some vertex $y$ in $P(n)$ and one in each of $P(m) \times\left\{y_{1}\right\}$ and $P(m) \times\left\{y_{2}\right\}$, where $y_{1}$ and $y_{2}$ are the opposite vertices of the two boundary edges with vertex $y$. Symmetrically for a simplex of type $\operatorname{Tr}_{n, 2}$. Thus, a simplex of type $\operatorname{Tr}_{m, 2} \cap \operatorname{Tr}_{n, 2}$ has vertices $(x, y),\left(x, y_{1}\right),\left(x, y_{2}\right),\left(x_{1}, y\right),\left(x_{2}, y\right)$, where $\left\langle x_{1}, x\right\rangle$ and $\left\langle x, x_{2}\right\rangle$ are two boundary edges in $P(m)$ and $\left\langle y_{1}, y\right\rangle$ and $\left\langle y, y_{2}\right\rangle$ are two boundary edges in $P(n)$. The 4-simplex hence contains the 4 triangles $\left\langle(x, y),\left(x, y_{1}\right),\left(x_{1}, y\right)\right\rangle$, $\left\langle(x, y),\left(x, y_{1}\right),\left(x_{2}, y\right)\right\rangle,\left\langle(x, y),\left(x, y_{2}\right),\left(x_{1}, y\right)\right\rangle$, and $\left\langle(x, y),\left(x, y_{2}\right),\left(x_{2}, y\right)\right\rangle$. There are no other triangles.

In a simplex of type $\operatorname{Tr}_{m, 2} \cap \operatorname{Tr}_{n, 1}$ or $\operatorname{Tr}_{m, 1} \cap \operatorname{Tr}_{n, 2}$, there exist precisely 2 triangles of the above form: A simplex of type $\operatorname{Tr}_{m, 2} \cap \operatorname{Tr}_{n, 1}$ has vertices $(x, y),\left(x, y_{1}\right),\left(x, y^{\prime}\right)$, $\left(x_{1}, y\right),\left(x_{2}, y\right)$, where $\left\langle x_{1}, x\right\rangle$ and $\left\langle x, x_{2}\right\rangle$ are the two vertices of two boundary edges in $P(m),\left\langle y_{1}, y\right\rangle$ is a boundary edge in $P(n)$, and $y^{\prime}$ is arbitrary. There are 2 triangles $\left\langle(x, y),\left(x, y_{1}\right),\left(x_{1}, y\right)\right\rangle$ and $\left\langle(x, y),\left(x, y_{1}\right),\left(x_{2}, y\right)\right\rangle$. If there were any more triangle, than the simplex would not be of type $\operatorname{Tr}_{n, 1}$ but of type $\operatorname{Tr}_{n, 2}$. Symmetrically for a simplex of type $\operatorname{Tr}_{m, 1} \cap \operatorname{Tr}_{n, 2}$.

In a simplex of type $\operatorname{Tr}_{m, 2} \cap B_{n}$ or $B_{m} \cap \operatorname{Tr}_{n, 2}$, there exist at least 2 triangles of the above form: A simplex of type $\operatorname{Tr}_{m, 2} \cap B_{n}$ has vertices $(x, y),\left(x_{1}, y_{1}\right),\left(x_{2}, y_{2}\right)$, $\left(x_{1}, y\right),\left(x_{2}, y\right)$, where $\left\{x_{1}, x_{2}\right\}$ is a boundary edge in $P(m)$, and $\left\{y_{1}, y\right\}$ and $\left\{y, y_{2}\right\}$ are the two vertices of two boundary edges in $P(n)$. There are now at least 2 triangles, namely $\left\langle\left(x_{1}, y_{1}\right),\left(x_{1}, y\right),\left(x_{2}, y\right)\right\rangle$ and $\left\langle\left(x_{1}, y\right),\left(x_{2}, y\right),\left(x_{2}, y_{2}\right)\right\rangle$. There may be more triangles (if $\left\langle x, x_{1}\right\rangle$ or $\left\langle x, x_{2}\right\rangle$ forms a boundary edge in $P(m)$ ). Symmetrically for a simplex of type $B_{m} \cap \operatorname{Tr}_{n, 2}$.

To prove the claim, it now remains to show that none of the above considered triangles has been counted twice. To see that, observe that a triangle in $\tau_{m} \times \tau_{n}$ is the 2-face of a unique 3-simplex in $P(m) \times \tau_{n}$ and a unique 3-simplex in $\tau_{m} \times P(n)$. Now, for every 4-simplex $\sigma$ and each of its triangles $t$ considered above, we have that if the triangle $t$ belongs to $\tau_{m} \times \tau_{n}$, then the 3-simplices in $P(m) \times \tau_{n}$ and $\tau_{m} \times P(n)$ which $t$ is a 2-face of, are 3-faces of $\sigma$. As a 4-simplex is completely determined by two of its 3 -faces, the claim follows.

In view of Claims 11 and 12, the number of 4-simplices with a 3-face of type Tr, type $A$, or type $B$ in $\partial(P(m) \times P(n))$ is equal to

$$
\begin{gathered}
t_{m, 1}+t_{m, 2}+a_{m}+b_{m}+t_{n, 1}+t_{n, 2}+a_{n}+b_{n}-t_{m, 1} \cap t_{n, 1}-t_{m, 1} \cap t_{n, 2}-t_{m, 1} \cap b_{n} \\
-t_{m, 2} \cap t_{n, 1}-t_{m, 2} \cap t_{n, 2}-t_{m, 2} \cap b_{n}-b_{m} \cap t_{n, 1}-b_{m} \cap t_{n, 2}-b_{m} \cap b_{n} .
\end{gathered}
$$

This expression can be rewritten as 


$$
\begin{aligned}
& \underbrace{\frac{1}{2} t_{m, 1}}_{=n(m-2)}+t_{m, 2}+\underbrace{\frac{1}{2} t_{n, 1}+t_{n, 2}}_{=m(n-2)}+\frac{1}{2} \underbrace{\left[t_{m, 1}-t_{m, 1} \cap t_{n, 1}-t_{m, 1} \cap t_{n, 2}-t_{m, 1} \cap b_{n}\right]}_{\geq 0} \\
& +\frac{1}{2} \underbrace{\left[t_{n, 1}-t_{m, 1} \cap t_{n, 1}-t_{m, 2} \cap t_{n, 1}-b_{m} \cap t_{n, 1}\right]}_{\geq 0} \\
& +\underbrace{a_{m}+\frac{1}{2} b_{m}}_{\geq 0}+\underbrace{a_{n}+\frac{1}{2} b_{n}}_{\geq \frac{1}{2} m(n-2)}+\frac{1}{2} \underbrace{\left[b_{m}-b_{m} \cap t_{n, 1}-b_{m} \cap t_{n, 2}-b_{m} \cap b_{n}\right]}_{\geq 0} \\
& +\frac{1}{2} \underbrace{\left[b_{n}-t_{m, 1} \cap b_{n}-t_{m, 2} \cap b_{n}-b_{m} \cap b_{n}\right]}_{\geq-m n} \\
& +\frac{1}{2} \underbrace{\left[-t_{m, 1} \cap t_{n, 2}-t_{m, 2} \cap t_{n, 1}-t_{m, 2} \cap b_{n}-b_{m} \cap t_{n, 2}-2 t_{m, 2} \cap t_{n, 2}\right]}_{\geq 0} \\
& \geq \frac{5}{2} m n
\end{aligned}
$$

where we have used the preliminary computations of Claims 13,14, and 15 as well as the inequalities preceding Claim 12 .

In fact, the statement of Theorem 10 can be improved as follows, since in its proof, we have really only counted simplices with a face of type $\operatorname{Tr}$, type $A$, or type $B$. Furthermore, the same partial estimates can be used to give a lower bound on the number of simplices with a face of type Tr.

Theorem 16 Let $T$ be any triangulation of $P(m) \times P(n)$. The number of 4-simplices of $T$ with a 3-face of type $\operatorname{Tr}$, type $A$, or type $B$ in $\partial(P(m) \times P(n))$ is greater than or equal to

$$
\frac{5}{2} m n-3(m+n) \text {. }
$$

Theorem 17 Let $T$ be a triangulation of $P(m) \times P(n)$. The number of 4-simplices of $T$ with a 3-face of type $\operatorname{Tr}$ in $\partial(P(m) \times P(n))$ is greater than or equal to

$$
\frac{3}{2} m n-2(m+n)
$$

Proof The number of 4-simplices considered in the statement of the theorem is equal to

$$
t_{m, 1}+t_{m, 2}+t_{n, 1}+t_{n, 2}-t_{m, 1} \cap t_{n, 1}-t_{m, 1} \cap t_{n, 2}-t_{m, 2} \cap t_{n, 1}-t_{m, 2} \cap t_{n, 2} .
$$


This expression can be rewritten as

$$
\begin{aligned}
& \underbrace{\frac{1}{2} t_{m, 1}+t_{m, 2}}_{=n(m-2)}+\underbrace{\frac{1}{2} t_{n, 1}+t_{n, 2}}_{=m(n-2)}+\frac{1}{2} \underbrace{\left[t_{m, 1}-t_{m, 1} \cap t_{n, 1}-t_{m, 1} \cap t_{n, 2}\right]}_{\geq 0} \\
& \quad+\frac{1}{2} \underbrace{\left[t_{n, 1}-t_{m, 1} \cap t_{n, 1}-t_{m, 2} \cap t_{n, 1}\right]}_{\geq 0} \\
& \quad+\frac{1}{2} \underbrace{\left[-t_{m, 1} \cap t_{n, 2}-t_{m, 2} \cap t_{n, 1}-2 t_{m, 2} \cap t_{n, 2}\right]}_{\geq-m n} \\
& \geq \frac{3}{2} m n-3(m+n) .
\end{aligned}
$$

\section{Lower Bounds}

Proposition 18 If $\sigma$ is a 4-simplex which contains a 3-face of type $\mathrm{Tr}$, then

$$
\left|\operatorname{Vol}_{4}(\sigma)\right| \leq \frac{1}{6}
$$

Proof If $\sigma$ contains a 3-face of type Tr, then by definition, it contains a 2-simplex of $P(m) \times\{*\}$ or $\{*\} \times P(n)$, so that three of its vertices (the vertices of this 2-simplex) have the same second (respectively first) coordinate, an the proposition then follows from Lemma 5.

Proposition 19 If $\sigma$ is a 4-simplex which contains a 3-face of type A or B, then

$$
\left|\operatorname{Vol}_{4}(\sigma)\right| \leq \frac{1}{3}
$$

In fact, if $\sigma$ is a 4-simplex which contains a 3-face of type $B$, then $\left|\operatorname{Vol}_{4}(\sigma)\right| \leq 1 / 6$.

Proof By symmetry, suppose without loss of generality that $\sigma$ has a 3-face of type $A_{m}$ or $B_{m}$. Thus, $\sigma$ has a 3-face $\alpha$ in some prism $P(m) \times \tau$. Denoting by $y, y^{\prime}$ the vertices of the edge $\tau$, observe furthermore that $\alpha$ has two vertices in $P(m) \times\{y\}$ and two in $P(m) \times\left\{y^{\prime}\right\}$. The proposition now follows from Lemma 6 .

Proof of Theorem 1 Let $T$ be a triangulation of $P(m) \times P(n)$, and let $\left[z_{T}\right]$ denote the corresponding affine cycle. By Proposition 4 , we have

$$
\operatorname{Vol}_{4}\left(\left[z_{T}\right]\right)=(m-2)(n-2) .
$$

By Theorem 17, there are at least

$$
\frac{3}{2} m n-2(m+n)
$$


4-simplices in $T$ which contain a 3-face of type Tr. Pick $(3 / 2) m n-2(m+n)$ of them (rounded down, for simplicity, if $m$ and $n$ are both odd). In view of Proposition 18, they contribute by a combinatorial volume of at most

$$
\frac{1}{6}\left(\frac{3}{2} m n-2(m+n)\right)
$$

By Theorem 16, there are at least

$$
m n-(m+n)
$$

further 4-simplices in $T$ which contain a 3-face of type Tr, type $A$, or type $B$. Pick $m n-(m+n)$ of them. In view of Propositions 18 and 19, they contribute by a combinatorial volume of at most

$$
\frac{1}{3}(m n-(m+n)) .
$$

Thus, the remaining volume is at least

$$
\begin{aligned}
& (m-2)(n-2)-\frac{1}{6}\left(\frac{3}{2} m n-2(m+n)\right)-\frac{1}{3}(m n-(m+n)) \\
& =\frac{5}{12} m n-\frac{4}{3}(m+n)+4 .
\end{aligned}
$$

Since the evaluation of the combinatorial volume cocycle on one 4-simplex is at most 2/3 (Lemma 8), we need at least

$$
\frac{3}{2}\left(\frac{5}{12} m n-\frac{4}{3}(m+n)+4\right)=\frac{5}{8} m n-2(n+m)+6
$$

more 4-simplices, so that

$$
T(m, n) \geq \frac{5}{2} m n-3(n+m)+\frac{5}{8} m n-2(n+m)+6=\frac{25}{8} m n-5(m+n)+6 .
$$

Proof of Theorem 2 If $n=4$, then exactly as in the proof of Theorem 1, the combinatorial volume of a triangulation of $P(m) \times P(4)$ is equal to $2(m-2)$. We can pick $4 m-8$ simplices which contain a 3 -face of type $\mathrm{Tr}$, which contribute by a combinatorial volume of at most $1 / 6(4 m-8)$. We can further pick $3 m-4$ simplices which contain a 3 -face of type $\operatorname{Tr}$, type $A$, or type $B$, which contribute by a combinatorial volume of at most $1 / 3(3 m-4)$. Thus the remaining volume is $1 / 3(m-4)$.

The difference now is that in view of Lemma 7 , the combinatorial volume of a 4-simplex with vertices in the vertices of $P(m) \times P(4)$ is at most $1 / 2$. Thus, we need at least

$$
2 \cdot \frac{1}{3}(m-4)
$$

more 4-simplices, so that

$$
T(m, 4) \geq 4 m-8+3 m-4+\frac{2}{3} m-\frac{8}{3}=\frac{23}{3} m-\frac{44}{3} .
$$


Acknowledgement I am grateful to Fransico Santos for his useful comments on preliminary versions of this paper.

\section{References}

1. Bowen, L., De Loera, J.A., Develin, M., Santos, F.: The Gromov norm of the product of two surfaces. Topology 44, 321-339 (2005)

2. Bucher-Karlsson, M.: The simplicial volume of closed manifolds covered by $\mathbb{H}^{2} \times \mathbb{H}^{2}$. J. Topology (2008). doi:10.1112/jtopol/jtn012

3. De Loera, J.A., Santos, F., Takeuchi, F.: Extremal properties for dissections of convex 3-polytopes. SIAM J. Discrete Math. 14(2), 143-161 (2001)

4. Sleator, D.D., Tarjan, R.E., Thurston, W.P.: Rotation distance triangulations and hyperbolic geometry. J. Am. Math. Soc. 1, 647-679 (1988)

5. Smith, W.: A lower bound for the simplexity of the $n$-cube via hyperbolic volumes. Combinatorics of polytopes. Eur. J. Combin. 21(1), 131-137 (2000) 